Pacific Journal of Mathematic 


\section{ON RAMSEY THEORY AND GRAPHICAL PARAMETERS}

\section{LiNDA LESNIAK-Foster AND JOHN RoBERTS}

A graph $G$ is said to have a factorization into the subgraphs $G_{1}, \cdots, G_{k}$ if the subgraphs are spanning, pairwise edge-disjoint, and the union of their edge sets equals the edge set of $G$. For a graphical parameter $f$ and positive integers $n_{1}, n_{2}, \cdots, n_{k}(k \geqq 1)$, the $f$-Ramsey number $f\left(n_{1}, n_{2}, \cdots, n_{k}\right)$ is the least positive integer $p$ such that for any factorization $K_{p}=\bigcup_{i=1}^{k} G_{i}$, it follows that $f\left(G_{\imath}\right) \geqq n_{\imath}$ for at least one $i$, $1 \leqq i \leqq k$. In the following, we present two results involving $f$-Ramsey numbers which hold for various vertex and edge partition parameters, respectively. It is then shown that the concept of $f$-Ramsey number can be generalized to more than one vertex partition parameter, more than one edge partition parameter, and combinations of vertex and edge partition parameters. Formulas are presented for these generalized $f$-Ramsey numbers and specific illustrations are given.

1. Introduction. A subgraph $H$ of a graph $G$ is called spanning if $H$ has the same vertex set as $G$. A graph $G$ is said to have a factorization into the subgraphs $G_{1}, G_{2}, \cdots, G_{k}$, written $G=\cup_{i=1}^{k} G_{l}$, if the subgraphs are spanning, pairwise edge-disjoint, and the union of their edge sets equals the edge set of $G$. It is permissible for a subgraph $G_{i}$ to be empty; i.e., have no edges.

Let $f$ be a graphical parameter, and let $n_{1}, n_{2}, \cdots, n_{k},(k \geqq 1)$ be positive integers. In [2], Chartrand and Polimeni defined the $f$-Ramsey number $f\left(n_{1}, n_{2}, \cdots, n_{k}\right)$ as the least positive integer $p$ such that for any factorization $K_{p}=\bigcup_{i=1}^{k} G_{i}$ of the complete graph of order $p$, it follows that $f\left(G_{i}\right) \geqq n_{i}$ for at least one subgraph $G_{i}, 1 \leqq i \leqq k$. If $\omega(G)$ is the maximum order among the complete subgraphs of $G$, then the $\omega$ Ramsey number is the ordinary Ramsey number (see [3; p. 16]) in $k$ variables.

The chromatic number $\chi(G)$ of a graph $G$ is the minimum number of colors which may be assigned to the vertices of $G$ so that adjacent vertices are assigned different colors. The vertex-arboricity $a(G)$ of $G$ is the minimum number of subsets into which the vertex set of $G$ may be partitioned so that each subset induces an acyclic subgraph. Chartrand and Polimeni [2] gave formulas for the $\chi$-Ramsey numbers and the $a$-Ramsey numbers. We present a result which holds for several "partition" parameters (including chromatic number and vertex- 
arboricity as special cases). Furthermore, it is shown that the concept of $f$-Ramsey number can be generalized to more than one parameter. Formulas are presented for these generalized $f$-Ramsey numbers, and specific illustrations are given involving chromatic number, edge chromatic number, vertex-arboricity, and arboricity.

2. Vertex partition parameters. A graphical property $\rho$ will be called co-hereditary if (1) every subgraph of a graph having property $\rho$ has property $\rho$ and (2) the graph consisting of disjoint graphs, each having property $\rho$, has property $\rho$.

Let $\nu$ be a graphical property which the trivial graph $K_{1}$ possesses. We define the vertex partition number $\nu(G)$ of a graph $G$ as the minimum number of subsets into which the vertex set of $G$ can be partitioned so that each subset induces a subgraph having property $\nu$. Clearly, $\nu(G)=1$ if and only if $G$ has property $\nu$. The limit $\lim \nu$ of a vertex partition parameter $\nu$ is defined as $\lim \nu=\lim _{n \rightarrow \infty} \nu\left(K_{n}\right)$, provided this $\operatorname{limit}$ exists. We write $\lim \nu=\infty$ if $\nu\left(K_{n}\right) \rightarrow \infty$ as $n \rightarrow \infty$. We assume that all properties $\nu$ under discussion are co-hereditary and that $\lim \nu=\infty$. It is a consequence of the definitions that $\nu(H) \leqq \nu(G)$ if $H$ is a subgraph of $G$, for such properties $\nu$.

For positive integers $n_{1}, n_{2}, \cdots, n_{k}$ and vertex partition parameter $\nu$, the $\nu$-Ramsey number $\nu\left(n_{1}, n_{2}, \cdots, n_{k}\right)$ is the least positive integer $p$ such that given any factorization $K_{p}=\bigcup_{i=1}^{k} G_{i}$, it follows that $\nu\left(G_{i}\right) \geqq n_{i}$ for at least one $i, 1 \leqq i \leqq k$. Since $\lim \nu=\infty$, for each $i, 1 \leqq i \leqq k$, there exists a positive integer $m_{i}$ such that $\nu\left(K_{m_{1}}\right) \geqq n_{l}$. Hence, since $\nu$ is cohereditary, $\quad \nu\left(n_{1}, n_{2}, \cdots, n_{k}\right)$ exists and is bounded above by $r\left(m_{1}, m_{2}, \cdots, m_{k}\right)$, the Ramsey number in the $k$ variables $m_{1}, m_{2}, \cdots, m_{k}$. We also note that $\nu\left(n_{1}, n_{2}, \cdots, n_{k}\right)$ is symmetric in $n_{1}, n_{2}, \cdots, n_{k}$.

There are properties $\nu$ which are not co-hereditary and for which $\lim \nu \neq \infty$ such that $\nu\left(n_{1}, n_{2}, \cdots, n_{k}\right)$ does not exist for certain positive integers $n_{1}, n_{2}, \cdots, n_{k}$. For example, if $\nu$ denotes the property of being connected, then $\nu(G)$ is the number of components of the graph $G$. Then $\nu(3,3)$ does not exist since for every positive integer $p$, there exists a factorization $K_{p}=G_{1} \cup G_{2}$ such that neither $G_{1}$ nor $G_{2}$ has more than two components.

For a vertex partition parameter $\nu$ and positive integer $k$, let $\bar{\nu}(k)$ denote the largest integer $p$ for which there exists a factorization $K_{p}=\bigcup_{i=1}^{k} G_{\imath}$ such that $\nu\left(G_{i}\right)=1$ for $i=1,2, \cdots, k$. Then we have the following lemma.

LEMMA 1. If $\nu$ is a vertex partition parameter for which $\lim \nu=\infty$ and the corresponding property $\nu$ is co-hereditary, then $\bar{\nu}(k)$ exists for every positive integer $k$. 
Proof. Since $\nu\left(K_{1}\right)=1$ and $K_{1}=\bigcup_{i=1}^{k} K_{1}$, it follows that $\bar{\nu}(k) \geqq 1$ for each positive integer $k$. Since $\lim \nu=\infty$, there exists a positive integer $p$ such that $\nu\left(K_{p}\right) \geqq 2$. Hence, if we consider an arbitrary factorization $K_{m}=\bigcup_{i=1}^{k} G_{l}$, where $m=r(p, p, \cdots, p)$ is the Ramsey number in $k$ variables, then $G_{i}$ contains $K_{p}$ as a subgraph for at least one $i, 1 \leqq i \leqq k$, say $i=j$. Therefore, $\nu\left(G_{l}\right) \geqq \nu\left(K_{p}\right) \geqq 2$, which implies that $\bar{\nu}(k)$ exists and, in fact, $\bar{\nu}(k)<m$.

We can now present a formula for any $\nu$-Ramsey number.

THEOREM 1. Let $n_{1}, n_{2}, \cdots, n_{k}(k \geqq 1)$ be positive integers, and let $\nu$ be a vertex partition parameter for which the corresponding property $\nu$ is co-hereditary and $\lim \nu=\infty$. Then

$$
\nu\left(n_{1}, n_{2}, \cdots, n_{k}\right)=1+\bar{\nu}(k) \cdot \prod_{i=1}^{k}\left(n_{i}-1\right) .
$$

Proof. If $n^{i}=1$ for some $i=1,2, \cdots, k$, then $\nu\left(n_{1}, n_{2}, \cdots, n_{k}\right)=1$ and the theorem follows. Thus, we assume that $n_{i} \geqq 2$ for each $i$, $1 \leqq i \leqq k$, and let

$$
p=1+\bar{\nu}(k) \cdot \prod_{i=1}^{k}\left(n_{i}-1\right)
$$

First, we verify the inequality $\nu\left(n_{1}, n_{2}, \cdots, n_{k}\right) \leqq p$. Assume that this is not the case. Then there exists a factorization $K_{p}=\bigcup_{i=1}^{k} G_{i}$ such that $\nu\left(G_{\imath}\right) \leqq n_{l}-1$ for $i=1,2, \cdots, k$. For each $G_{\imath}, i=1,2, \cdots, k$, let the vertex set $V\left(G_{i}\right)$ be partitioned into $\nu\left(G_{i}\right)$ classes so that for each " $\nu$-class" $\propto, \nu(\langle\propto\rangle)=1$, where $\langle\propto\rangle$ denotes the subgraph induced by the class $\propto$. Then $G_{1}$ has a $\nu$-class $\propto_{1}$ containing at least $1+$ $\bar{\nu}(k) \Pi_{i=2}^{k}\left(n_{i}-1\right)$ vertices, $G_{2}$ has a $\nu$-class $\propto_{2}$ containing at least $1+\bar{\nu}(k) \cdot \prod_{i=3}^{k}\left(n_{t}-1\right)$ vertices of $\propto_{1}$, and, in general, for $1 \leqq l<k$, if $\propto_{1}, \propto_{2}, \cdots, \propto_{l}$ are $\nu$-classes, respectively, of $G_{1}, G_{2}, \cdots, G_{l}$ for which

$$
\left|\bigcap_{i=1}^{l} \propto_{l}\right| \geqq 1+\bar{\nu}(k) \cdot \prod_{i=l+1}^{k}\left(n_{i}-1\right),
$$

then $G_{l+1}$ has a $\nu$-class $\propto_{l+1}$ such that

$$
\left|\bigcap_{i=1}^{l+1} \propto_{i}\right| \geqq 1+\bar{\nu}(k) \cdot \prod_{i=l+2}^{k}\left(n_{i}-1\right) .
$$

Hence, each $G_{i}, 1 \leqq i \leqq k$, has a $\nu$-class $\propto_{i}$ such that $\left|\cap_{i=1}^{k} \propto_{i}\right| \geqq$ $1+\bar{\nu}(k)$. Let $\mathcal{U}$ be a set of $1+\bar{\nu}(k)$ vertices in $\bigcap_{i=1}^{k} \propto$, and define $H_{i}$ to be the subgraph in $G_{i}$ induced by $u$ for $i=1,2, \cdots, k$. Then 


$$
K_{1+\bar{\nu}(k)}=H_{1} \cup H_{2} \cup \cdots \cup H_{k}
$$

where $\nu\left(H_{i}\right)=1$ for $1 \leqq i \leqq k$ since $\nu$ is co-hereditary. This, however, is impossible. Therefore, $\nu\left(n_{1}, n_{2}, \cdots, n_{k}\right) \leqq p$.

In order to show that $\nu\left(n_{1}, n_{2}, \cdots, n_{k}\right)=p$, it suffices to exhibit a factorization $K_{p-1}=\bigcup_{i=1}^{k} G_{i}$ such that $\nu\left(G_{i}\right) \leqq n_{l}-1$ for $i=$ $1,2, \cdots, k$. Let $r=\prod_{i=1}^{k}\left(n_{t}-1\right)$, and consider $r$ pairwise disjoint copies of $K_{\bar{\nu}(k)}$, labeled $K_{\bar{\nu}(k)}^{1}, K_{\bar{\nu}(k)}^{2}, \ldots, K_{\bar{\nu}(k)}^{r}$. By definition of $\bar{\nu}(k)$, there exists a factorization $K_{\bar{\nu}(k)}=\bigcup_{i=1}^{k} F_{i}$ such that $\nu\left(F_{i}\right)=1$ for $i=1,2, \cdots, k$. We denote by $F_{i l}$ the $F_{1}$ contained in $K_{\bar{\nu}(k)}^{l}, l=1,2, \cdots, r$ and $i=1,2, \cdots, k$. With each of the $r k$-tuples $\left(c_{1}, c_{2}, \cdots, c_{k}\right), 1 \leqq c_{j} \leqq n_{j}-1$ and $1 \leqq j \leqq k$, we identify a complete graph $K_{\bar{\nu}(k)}^{l}, l=1,2, \cdots, r$, in such a way that the identification is one-to-one. Then, for each $i=1,2, \cdots, k$ and $l=$ $1,2, \cdots, r$, we associate with $F_{l l}$ the $k$-tuple identified with $K_{\bar{\nu}(k)}^{\prime}$. Let the graph $G_{i}(i=1,2, \cdots, k)$ consist of the graphs $F_{i 1}, F_{i 2}, \cdots, F_{i r}$ where a vertex of $F_{t s}$ is adjacent to each vertex of $F_{t t}$ if and only if the $i$ th coordinate is the first coordinate in which their associated $k$-tuples differ. It then follows that $K_{p-1}=\bigcup_{i=1}^{k} G_{i}$. For each $i=1,2, \cdots, k$, define $V_{b, j}$ to consist of the set of all vertices $v$ such that $v$ is a vertex of an $F_{i l}$ whose associated $k$-tuple $\left(c_{1}, c_{2}, \cdots, c_{k}\right)$ has $c_{\imath}=j ; j=1,2, \cdots, n_{l}-1$. Then $\left\{V_{i, 1}, V_{i, 2}, \cdots, V_{i, n_{i}-1}\right\}$ is a partition of $V\left(G_{i}\right)$ for which the subgraph $\left\langle V_{t, j}\right\rangle$ consists of $r /\left(n_{i-1}\right)$ pairwise disjoint copies of $F_{i}, J=1,2, \cdots, n_{l}-1$. Hence, $\nu\left(V_{i, j}\right)=1$ for each such $j$, which implies that $\nu\left(G_{\imath}\right) \leqq n_{i-1}$ for $i=1,2, \cdots, k$.

For the chromatic number $\chi$, it follows that $\bar{\chi}(k)=1$ for all $k \geqq 1$. Hence, we obtain an immediate corollary.

Corollary 1a. (Chartrand and Polimeni [2]). If $n_{1}, n_{2}, \cdots, n_{k}$ are positive integers, then

$$
\chi\left(n_{1}, n_{2}, \cdots, n_{k}\right)=1+\prod_{i=1}^{k}\left(n_{i}-1\right)
$$

The edge-arboricity $a_{1}(G)$ of a graph $G$ is the minimum number of subsets in a partition of the edge set of $G$ such that each subset induces an acyclic subgraph. For the vertex-arboricity $a(G)$ of $G$, we have another corollary.

Corollary 1b. (Chartrand and Polimeni [2]). If $n_{1}, n_{2}, \cdots, n_{k}$ are positive integers, then

$$
a\left(n_{1}, n_{2}, \cdots, n_{k}\right)=1+2 k \cdot \prod_{i=1}^{k}\left(n_{i}-1\right) .
$$


Proof. Again, it suffices to evaluate $\bar{a}(k)$. First, we observe that if there is a factorization $K_{p}=\bigcup_{i=1}^{k} G_{i}$, where $a\left(G_{\imath}\right)=1$ for $i=1,2, \cdots, k$, then $k \geqq a_{1}\left(K_{p}\right)=\{p / 2\}$. Since $p=2 k$ is the largest such integer, we have $\bar{a}(k)=2 k$ and the desired result.

As one further illustration of Theorem 1, we consider the 2chromatic number $\chi^{(2)}(G)$ of a graph $G$ (see 1), defined as the least number of subsets in any partition of $V(G)$ such that the subgraph induced by each subset contains no path of length two. Also, we define the edge chromatic number $\chi_{1}(G)$ of $G$ as the least number of colors needed to color the edges of $G$ so that adjacent edges are colored differently.

COROllary 1c. For positive integers $n_{1}, n_{2}, \cdots, n_{k}$,

$$
\chi^{(2)}\left(n_{1}, n_{2}, \cdots, n_{k}\right)=1+2\left\{\frac{k}{2}\right\} \cdot \prod_{i=1}^{k}\left(n_{t}-1\right) .
$$

Proof. To determine $\bar{\chi}^{(2)}(k)$, it is equivalent to determine the largest integer $n$ such that $\chi_{1}\left(K_{n}\right) \leqq k$. Since $\chi_{1}\left(K_{p}\right)=p$ if $p$ is odd and $\chi_{1}\left(K_{p}\right)=p-1$ if $p$ is even, it follows that $n=2\{k / 2\}$, which gives the desired result.

The concept of the $\nu$-Ramsey number can be generalized. Let $\nu_{1}, \nu_{2}, \cdots, \nu_{k}$ be vertex partition parameters where again we assume the corresponding properties are co-hereditary and $\lim \nu_{t}=\infty$ for each $i$, $1 \leqq i \leqq k$. Then we define the $\left(\nu_{l}\right)_{1}^{k}$-Ramsey number $\left(\nu_{l}\right)_{1}^{k}\left(n_{1}, n_{2}, \cdots, n_{k}\right)$ as the least positive integer $p$ such that given any factorization $K_{p}=$ $\bigcup_{i=1}^{k} G_{t}$, it follows that $\nu_{t}\left(G_{t}\right) \geqq n_{t}$ for at least one $i, 1 \leqq i \leqq k$. Following an earlier argument we note that $\left(\nu_{i}\right)_{1}^{k}\left(n_{1}, n_{2}, \cdots, n_{k}\right)$ exists since each $\nu_{l}$ is co-hereditary and $\lim \nu_{\imath} \equiv \infty$. In this case, we do not have symmetry in the $k$-variables $n_{1}, n_{2}, \cdots, n_{k}$; however, it does follow that

$$
\left(\nu_{i_{j}}\right)_{j=1}^{k}\left(n_{t_{1}}, n_{t 2}, \cdots, n_{t k}\right)=\left(\nu_{t}\right)_{1}^{k}\left(n_{1}, n_{2}, \cdots, n_{k}\right)
$$

where $i_{1}, i_{2}, \cdots, i_{k}$ is any permutation of $1,2, \cdots, k$.

For vertex partition parameters $\nu_{1}, \nu_{2}, \cdots, \nu_{k}$, we define $\left(\bar{\nu}_{i}\right)_{1}^{k}(k)=$ $(\bar{\nu})_{1}^{k}$ to be the largest integer $p$ such that there exists a factorization $K_{p}=\bigcup_{i=1}^{k} G_{i}$ with $\nu_{t}\left(G_{i}\right)=1$ for $i=1,2, \cdots, k$. Using an argument similar to that given in Lemma 1 , one can show that $\left(\bar{\nu}_{1}\right)_{1}^{k}$ exists, and moreover, a technique analogous to that employed in the proof of Theorem 1 can be used to verify the following generalization of Theorem 1. 
THEOREM 2. Let $n_{1}, n_{2}, \cdots, n_{k}(k \geqq 1)$ be positive integers, and let $\nu_{1}, \nu_{2}, \cdots, \nu_{k}$ be vertex partition parameters for which the corresponding properties $\nu_{i}$ are co-hereditary and $\lim \nu_{i}=\infty$ for $1 \leqq i \leqq k$. Then

$$
\left(\nu_{i}\right)_{1}^{k}\left(n_{1}, n_{2}, \cdots, n_{k}\right)=1+\left(\bar{\nu}_{1}\right)_{1}^{k} \cdot \prod_{i=1}^{k}\left(n_{i}-1\right) .
$$

By setting $\nu_{\imath}=\nu$ for $i=1,2, \cdots, k$ in the statement of Theorem 2, we obtain Theorem 1. We present two specific illustrations of Theorem 2 .

COROllary 2a. Let $n_{1}, n_{2}, \cdots, n_{k}(k \geqq 1)$ be positive integers, and let $\nu_{1}, \nu_{2}, \cdots, \nu_{k}$ be parameters such that $\nu_{t}=a$ for $1 \leqq i \leqq t$, where $1 \leqq t \leqq$ $k$, and $\nu_{\imath}=\chi$ for all other $\nu_{i}$. Then

$$
\left(\nu_{i}\right)_{1}^{k}\left(n_{1}, n_{2}, \cdots, n_{k}\right)=1+2 t \cdot \prod_{i=1}^{k}\left(n_{i}-1\right) .
$$

Proof. In this case, $\left(\bar{\nu}_{t}\right)_{1}^{k}$ is the largest integer $p$ such that there exists a factorization $K_{p}=\bigcup_{i=1}^{k} G_{i}$ with $a\left(G_{i}\right)=1$ for $1 \leqq i \leqq t$ and $\chi\left(G_{i}\right)=1$ for all other $i$ such that $i \leqq k$. This is clearly equal to $\bar{a}(t)$, which has the value $2 t$.

Similarly, since $\bar{\chi}^{(2)}(t)=2\{t / 2\}$ for each positive integer $t$, we have the following.

COROllary $2 \mathrm{~b}$. Let $n_{1}, n_{2}, \cdots, n_{k}(k \geqq 1)$ be positive integers, and let $\nu_{1}, \nu_{2}, \cdots, \nu_{k}$ be parameters such that $\nu_{i}=\chi^{(2)}$ for $1 \leqq i \leqq t$, where $1 \leqq t \leqq k$ and $\nu_{i}=\chi$ for all other $\nu_{i}$. Then

$$
\left(\nu_{i}\right)_{1}^{k}\left(n_{1}, n_{2}, \cdots, n_{k}\right)=1+2\{t / 2\} \cdot \prod_{i=1}^{k}\left(n_{i}-1\right) .
$$

3. Edge partition parameters. Let $\epsilon$ denote a graphical property which the graph $K_{2}$ possesses. We then define the edge partition number $\epsilon(G)$ of a nonempty graph $G$ as the least number of elements $E_{l}$ in a partition of the edge set $E(G)$ of $G$ such that each induced subgraph $E_{i}$ has property $\epsilon$. It is clearly equivalent to say that $\epsilon(G)$ is the minimum positive $k$ for which there exists a factorization $G=\bigcup_{i=1}^{k} G_{i}$ such that $\epsilon\left(G_{i}\right)=1$ for $i=1,2, \cdots, k$. For an empty graph $G$, we define $\epsilon(G)=0$. In this section, we shall henceforth assume that $\epsilon$ is a co-hereditary property (so that $H$ is a subgraph of $G$ implies that

$$
\epsilon(H) \leqq \epsilon(G) \text { ), and that } \lim \epsilon=\infty \text { (i.e., } \lim _{n \rightarrow \infty} \epsilon\left(K_{n}\right)=\infty \text { ). }
$$


Our next lemma, presented without proof, is an immediate consequence of the definitions for edge partition number and factorization.

LEMMA 2. If $G=\bigcup_{i=1}^{k} G_{i}$ and $\epsilon$ is an edge partition parameter, then

$$
\epsilon(G) \leqq \sum_{i=1}^{k} \epsilon\left(G_{i}\right)
$$

Let $\epsilon$ be an edge partition parameter, and let $n_{1}, n_{2}, \cdots, n_{k}(k \geqq 1)$ be nonnegative integers. The $\epsilon$-Ramsey number $\epsilon\left(n_{1}, n_{2}, \cdots, n_{k}\right)$ is the least positive integer $p$ such that for any factorization $K_{p}=\bigcup_{i=1}^{k} G_{i}$, it follows that $\epsilon\left(G_{i}\right) \geqq n_{i}$ for at least one $i, 1 \leqq i \leqq k$. If $n_{i}=0$ for some $i$, $1 \leqq i<k$, then clearly $\epsilon\left(n_{1}, n_{2}, \cdots, n_{k}\right)=1$; hence, we henceforth assume that $n_{\imath}>0$ for $i=1,2, \cdots, k$. Using an argument analogous to those used earlier, one can verify that $\epsilon\left(n_{1}, n_{2}, \cdots, n_{k}\right)$ exists since $\epsilon$ is co-hereditary and $\lim \epsilon=\infty$. In this case also, $\epsilon\left(n_{1}, n_{2}, \cdots, n_{k}\right)$ is symmetric in $n_{1}, n_{2}, \cdots, n_{k}$.

THEOREM 3. Let $n_{1}, n_{2}, \cdots, n_{k}(k \geqq 1)$ be positive integers, and let $\epsilon$ be an edge partition parameter such that the corresponding property $\epsilon$ is co-hereditary and $\lim \epsilon=\infty$. Then $\epsilon\left(n_{1}, n_{2}, \cdots, n_{k}\right)=N$ where

$$
N=1+\max \left\{p \mid \epsilon\left(K_{p}\right) \leqq \sum_{i=1}^{k}\left(n_{i}-1\right)\right\}
$$

Proof. Since $\lim \epsilon=\infty$ and $\epsilon\left(K_{1}\right)=0, \quad N$ exists and $N \geqq$ 2. Without loss of generality, we assume that $n_{1} \leqq n_{2} \leqq \cdots \leqq n_{k}$. The theorem clearly follows if $n_{k}=1$. Thus we assume that $n_{k} \geqq 2$.

First, we establish the inequality $\epsilon\left(n_{1}, n_{2}, \cdots, n_{k}\right) \leqq N$. Let $\bigcup_{i=1}^{k} G_{i}$ be a factorization of $K_{N}$. It follows from Lemma 2 and the definition of $N$ that

$$
\sum_{i=1}^{k} \epsilon\left(G_{i}\right) \geqq \epsilon\left(K_{N}\right)>\sum_{i=1}^{k}\left(n_{t}-1\right)
$$

Thus, $\epsilon\left(G_{\imath}\right) \geqq n_{i}$ for at least one $i, 1 \leqq i \leqq k$.

In order to show that $\epsilon\left(n_{1}, n_{2}, \cdots, n_{k}\right) \geqq N$, we exhibit a factorization $K_{N-1}=\bigcup_{i=1}^{k} G_{i}$, where $\epsilon\left(G_{i}\right) \leqq n_{i}-1$ for $1 \leqq i \leqq k$. Let $m$ be the least integer such that $n_{m} \geqq 2$. By the way in which $N$ and $m$ were chosen,

$$
\epsilon\left(K_{N-1}\right) \leqq \sum_{i=1}^{k}\left(n_{i}-1\right)=\sum_{i=m}^{k}\left(n_{i}-1\right)
$$

This implies that there exists a factorization 


$$
K_{N-1}=\bigcup_{i=m}^{k} \bigcup_{j=1}^{n_{i}-1} H_{j}^{\prime}, \text { where } \epsilon\left(H_{\jmath}^{\prime}\right) \leqq 1
$$

( $m \leqq i \leqq k$ and $\left.1 \leqq j \leqq n_{t}-1\right)$. For $m \leqq i \leqq k$, let $G_{t}=\bigcup_{j=1}^{n_{i}-1} H_{r}^{\iota}$. For $1 \leqq i \leqq m-1$ (if such $i$ exists), let $G_{t}=\bar{K}_{N-1}$, the complement of $K_{N-1}$. Then $K_{N-1}=\bigcup_{i=1}^{k} G_{i}$, where $\epsilon\left(G_{i}\right) \leqq n_{i}-1$ for $1 \leqq i \leqq k$.

Using the fact that the edge chromatic number $\chi_{1}\left(K_{p}\right)$ of a nontrivial complete graph $K_{p}$ is $p$ if $p$ is odd and $p-1$ if $p$ is even, we obtain the following corollary.

COROllary 3a. Let $n_{1}, n_{2}, \cdots, n_{k}$ be positive integers. If $n_{1}=n_{2}=$ $\cdots=n_{k}=1$, then $\chi_{1}\left(n_{1}, n_{2}, \cdots, n_{k}\right)=2$. Otherwise, $\chi_{1}\left(n_{1}, n_{2}, \cdots, n_{k}\right)=$ $2[(L+1) / 2]+1$ where $L=\sum_{\imath=1}^{k}\left(n_{t}-1\right)$.

Corollary 3b. Let $n_{1}, n_{2}, \cdots, n_{k}$ be positive integers, and let $a_{1}$ denote the (edge) arboricity parameter. If $n_{1}=n_{2}=\cdots=n_{k}=1$, then $a_{1}\left(n_{1}, n_{2}, \cdots, n_{k}\right)=2$. Otherwise,

$$
a_{1}\left(n_{1}, n_{2}, \cdots, n_{k}\right)=1+2 \sum_{i=1}^{k}\left(n^{\prime}-1\right)
$$
$\{p / 2\}$.

Proof. The result follows from the fact that for $p \geqq 2, a_{1}\left(K_{p}\right)=$

Let $n_{1}, n_{2}, \cdots, n_{k}$ be nonnegative integers, and let $\epsilon_{1}, \epsilon_{2}, \cdots, \epsilon_{k}$ be edge partition parameters where again we assume the corresponding properties are co-hereditary and $\lim \epsilon_{i}=\infty$ for each $i, 1 \leqq i \leqq k$. Then we may define the $\left(\epsilon_{i}\right)_{1}^{k}$-Ramsey number $\left(\epsilon_{t}\right)_{1}^{k}\left(n_{1}, n_{2}, \cdots, n_{k}\right)$ as the least positive integer $p$ such that for any factorization $K_{p}=\bigcup_{i=1}^{k} G_{l}$, it follows that $\epsilon_{l}\left(G_{l}\right) \geqq n_{\imath}$ for at least one $i$ where $1 \leqq i \leqq k$. Again there is no loss of generality in assuming each $n_{i}>0$ since $\left(\epsilon_{i}\right)_{1}^{k}\left(n_{1}, n_{2}, \cdots, n_{k}\right)=1$ if $n_{i}=0$ for some $i, 1 \leqq i \leqq k$. If we let $M$ denote the largest integer $p$ for which there exists a factorization $K_{p}=\bigcup_{i=1}^{k} G_{i}$ such that $\epsilon_{i}\left(G_{i}\right) \leqq n_{i}-1$ for $i=1,2, \cdots, k$, then it follows that $M$ exists and that $\left(\epsilon_{1}\right)_{1}^{k}\left(n_{1}, n_{2}, \cdots, n_{k}\right)=$ $1+M$; however, it is not possible to give such a compact expression for $\left(\epsilon_{l}\right)_{1}^{k}\left(n_{1}, n_{2}, \cdots, n_{k}\right)$ as for one edge partition parameter (Theorem 3 ) or $k$ vertex partition parameters.

As an illustration of the foregoing, we consider $\left(a_{1}, \chi_{1}\right)(m, n)$, for positive integers $m \geqq 2$ and $n \geqq 2$, defined as the least integer $p$ such that for any factorization $K_{p}=G_{1} \cup G_{2}$, either $a_{1}\left(G_{1}\right) \geqq m$ or $\chi_{1}\left(G_{2}\right) \geqq n$.

First we show that for every two such positive integers, we have $\left(a_{1}, \chi_{1}\right)(m, n) \leqq 2 m+n-2$. If this is not the case, then there exists a factorization $K_{2 m+n-2}=G_{1} \cup G_{2}$ such that $a_{1}\left(G_{1}\right) \leqq m-1$ and $\chi_{1}\left(G_{2}\right) \leqq$ 
$n-1$. This implies that $G_{1}$ has at most $(m-1)(2 m+n-3)$ edges while $G_{2}$ has at most $(n-1) \cdot[(2 m+n-2) / 2]$ edges. However, this implies that $K_{2 m+n-2}$ has less than $(2 m+n-2)(2 m+n-3) / 2$ edges, thereby producing a contradiction.

Next, we note for every positive integer $m \geqq 2$ and every odd positive integer $n \geqq 3$, that $\left(a_{1}, \chi_{1}\right)(m, n)=2 m+n-2$. Here, it suffices to produce a factorization $K_{2 m+n-3}=F_{1} \cup F_{2}$ for which $a_{1}\left(F_{1}\right) \leqq m-1$ and $\chi_{1}\left(F_{2}\right) \leqq n-1$. Since $2 m+n-3$ is even, there exists a factorization $K_{2 m+n-3}=\bigcup_{i=1}^{k} P_{i}$, where $k=(2 m+n-3) / 2$ and $P_{\imath}$ is a spanning path, $i=1,2, \cdots, k$ (see [3,p. 91]). Let $F_{1}=\bigcup_{i=1}^{m=1} P_{i}$. For $m \leqq i \leqq k$, we can write $P_{1}=P_{1,1} \cup P_{1,2}$ where no two edges of $P_{i, j}, j=1,2$, have adjacent edges. If we let $F_{2}=\bigcup_{l=m}^{k}\left[P_{t, 1} \cup P_{t, 2}\right]$, then we see that $\chi_{1}\left(F_{2}\right) \leqq$ $n-1$. Since $a_{1}\left(F_{1}\right) \leqq m-1$, we have a suitable factorization.

Based on the previous observations, we offer the following conjecture.

COnJecture. For every two positive integers $m \geqq 2$ and $n \geqq 2$,

$$
\left(a_{1}, \chi_{1}\right)(m, n)=2 m+n-2 \text {. }
$$

4. Vertex and edge partition parameters. Let $k=$ $k_{1}+k_{2}$, where $k_{1}, i=1,2$, is a positive integer. Denote by $\rho_{1}, \rho_{2}, \cdots, \rho_{k_{1}}$ vertex partition parameters, and denote by $\rho_{k_{1}+1}, \rho_{k_{1}+2}, \cdots, \rho_{k}$ edge partition parameters, for which the corresponding properties $\rho_{i}(1 \leqq i \leqq$ $k$ ) are co-hereditary and $\lim \rho_{\imath}=\infty$. For positive integers $n_{1}, n_{2}, \cdots, n_{k_{1}}$ and nonnegative integers $n_{k_{1}+1}, n_{k_{1}+2}, \cdots, n_{k}$, we define the $\left(\rho_{i}\right)_{1}^{k}$-Ramsey number $\left(\rho_{1}\right)_{1}^{k}\left(n_{1}, n_{2}, \cdots, n_{k}\right)$ as the least positive integer $\rho$ such that for any factorization $K_{p}=\bigcup_{i=1}^{k} G_{i}, \rho_{i}\left(G_{i}\right) \geqq n_{i}$ for at least one $i, 1 \leqq i \leqq$ $k$. Here we also have that if $i_{1}, i_{2}, \cdots, i_{k}$ is a permutation of $1,2, \cdots, k$, then

$$
\left(\rho_{i_{j}}\right)_{J=1}^{k}\left(n_{i_{1}}, n_{t}, \cdots, n_{i k}\right)=\left(\rho_{i}\right)_{1}^{k}\left(n_{1}, n_{2}, \cdots, n_{k}\right) .
$$

Let $\overline{\left(\rho_{i}\left(n_{i}\right)\right)_{1}^{k}}$ denote the largest positive integer $p$ for which there exists a factorization $K_{p}=\bigcup_{i=1}^{k} G_{i}$, where $\rho_{i}\left(G_{i}\right) \leqq n_{t}$ for $i=1,2, \cdots, k$. An argument similar to that used in the proof of Lemma 1 guarantees the existence of $\overline{\left(\rho_{t}\left(n_{t}\right)\right)_{1}^{k}}$ and a straightforward extension of the proof of Theorem 1 can be used to demonstrate the following result.

THEOREM 4. Let $k_{1}$ and $k_{2}$ be positive integers, where $k=$ $k_{1}+k_{2}$. For $i=1,2, \cdots, k_{1}$, let $\rho_{i}$ be a vertex partition parameter and for $i=k_{1}+1, k_{1}+2, \cdots, k$, let $\rho_{\imath}$ be an edge partition parameter such that the corresponding properties $\rho_{i}, 1 \leqq i \leqq k$, are co-hereditary and $\lim \rho_{i}=\infty$, $1 \leqq i \leqq k$. Then for positive integers $n_{1}, n_{2}, \cdots, n_{k}$, 


$$
\left(\rho_{i}\right)_{1}^{k}\left(n_{1}, n_{2}, \cdots, n_{k}\right)=1+\overline{\left(\rho_{l}\left(m_{\imath}\right)\right)_{1}^{k}} \cdot \prod_{i=1}^{k_{1}}\left(n_{i}-1\right),
$$

where $m_{\imath}=1$ for $1 \leqq i \leqq k_{1}$, and $m_{i}=n_{\imath}=1$ for $k_{\imath}+1 \leqq i \leqq k$.

As an illustration of Theorem 4, we present the following corollary.

COROLlARY 4a. Let $k=k_{1}+k_{2}$, where $k_{1}$ and $k_{2}$ are positive integers, and let $n_{1}, n_{2}, \cdots, n_{k}$ be positive integers. If $\rho_{i}=\chi$ for $1 \leqq i \leqq k_{1}$ and $\rho_{\imath}=\chi_{1}$ for $k_{1}+1 \leqq i \leqq k$, then

$$
\left(\rho_{l}\right)_{1}^{k}\left(n_{1}, n_{2}, \cdots, n_{k}\right)=1+\left[\chi_{1}\left(n_{k_{1}+1}, n_{k_{1}+2}, \cdots, n_{k}\right)-1\right] \cdot \prod_{i=1}^{k_{1}}\left(n_{i}-1\right) .
$$

Proof. By Theorem 4, it suffices to evaluate $\overline{\left(\rho_{i}\left(m_{i}\right)\right)_{1}^{k}}$, where $m_{\mathrm{t}}=1$ for $1 \leqq i \leqq k_{1}$ and $m_{i}=n_{\imath}-1$ for $k_{1}+\underline{1 \leqq i \leqq k}$. However, since $\rho_{i}=\chi$ for $1 \leqq i \leqq k_{1}$, it suffices to consider $\overline{\left(\rho_{t}\left(m_{\imath}\right)\right)_{k_{1}+1}^{k}}$, which equals $\chi_{1}\left(n_{k_{1}+1}\right.$, $\left.n_{k_{1}+2}, \cdots, n_{k}\right)-1$.

In a similar manner, we obtain the following result concerning chromatic number $\chi$ and edge arboricity $a_{1}$.

COROLlARY 4b. Let $k=k_{1}+k_{2}$, where $k_{1}$ and $k_{2}$ are positive integers, and let $n_{1}, n_{2}, \cdots, n_{k}$ be positive integers. If $\rho_{i}=\chi$ for $1 \leqq i \leqq k_{1}$ and $\rho_{\imath}=a_{1}$ for $k_{1}+1 \leqq i \leqq k$, then

$$
\left(\rho_{i}\right)_{1}^{k}\left(n_{1}, n_{2}, \cdots, n_{k}\right)=1+\left[a_{1}\left(n_{k_{1}+1}, n_{k_{1}+2}, \cdots, n_{k}\right)-1\right] \cdot \prod_{i=1}^{k_{1}}\left(n^{i}-1\right) .
$$

\section{REFERENCES}

1. G. Chartrand, D. Geller, and S. Hedetniemi, A generalization of the chromatic number, Proc. Comb. Phil. Soc., 64 (1968), 265-271.

2. G. Chartrand and A. D. Polimeni, Ramsey theory and chromatic numbers, Pacific J. Math., (to appear).

3. F. Harary, Graph Theory, Addison-Wesley, Reading, Mass. (1969).

Received June 9, 1975 and in revised form July 23, 1976.

Louisiana State University

AND

UNIVERSITY OF LOUISVILLE

LOUISVILLE, KY 40208 



\section{Pacific Journal of Mathematics}

Vol. 68, No. 1

March, 1977

Richard Julian Bagby, On $L^{p}, L^{q}$ multipliers of Fourier transforms . .......

Robert Beauwens and Jean-Jacques Van Binnebeek, Convergence theorems in

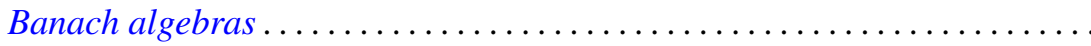

James Cyril Becker, Skew linear vector fields on spheres in the stable

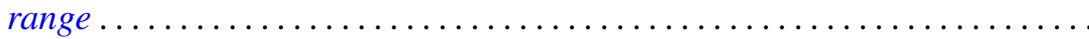

Michael James Beeson, Continuity and comprehension in intuitionistic formal

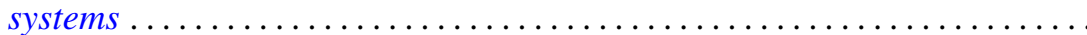

James K. Deveney, Generalized primitive elements for transcendental field

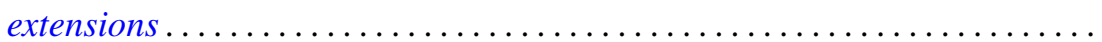

Samuel S. Feder, Samuel Carlos Gitler and K. Y. Lam, Composition properties

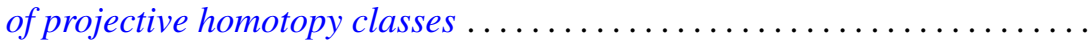

Nathan Jacob Fine, Tensor products of function rings under composition ......

Benno Fuchssteiner, Iterations and fixpoints . . . . . . . . . . . . . .

Wolfgang H. Heil, On punctured balls in manifolds

Shigeru Itoh, A random fixed point theorem for a multivalued contraction

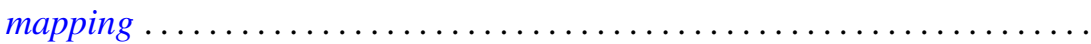

Nicolas P. Jewell, Continuity of module and higher derivations . . . . . . ......

Roger Dale Konyndyk, Residually central wreath products . . . . . . . . . . .

Linda M. Lesniak and John A. Roberts, On Ramsey theory and graphical

parameters.

Vo Thanh Liem, Some cellular subsets of the spheres.

Dieter Lutz, A perturbation theorem for spectral operators

P. H. Maserick, Moments of measures on convex bodies ... . . .

Stephen Joseph McAdam, Unmixed 2-dimensional local domains . .

D. B. McAlister and Norman R. Reilly, E-unitary covers for inverse semigroups...

William H. Meeks, III and Julie Patrusky, Representing codimension-one

homology classes by embedded submanifolds . . .

Premalata Mohapatro, Generalised quasi-Nörlund summability . .

Takahiko Nakazi, Superalgebras of weak-*Dirichlet algebras .

Catherine Louise Olsen, Norms of compact perturbations of operators .

William Henry Ruckle, Absolutely divergent series and isomorphism of

subspaces. II.

Bernard Russo, On the Hausdorff-Young theorem for integral operators .

Arthur Argyle Sagle and J. R. Schumi, Anti-commutative algebras and

homogeneous spaces with multiplications ............

Robert Evert Stong, Stiefel-Whitney classes of manifolds .

D. Suryanarayana, On a theorem of Apostol concerning Möbius functions of

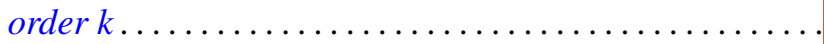

Yoshio Tanaka, On closedness of $C$ - and $C^{*}$-embeddings . . 